\title{
The longitude problem from the 1700s to today: An international and general education physics course
}

Thomas J. Bensky

Department of Physics, California Polytechnic State University, San Luis Obispo, California 93407

\begin{abstract}
For instructors wishing to use physics as part of an international or general education course, the framework for a course based on the "longitude problem" from the 1700s is described. The longitude problem is teeming with basic principles of physics and astronomy, which makes it ideal for a nonscience-major-based college-level course. This paper summarizes the longitude problem in the context of conceptual physics and astronomy and outlines an appropriate curriculum. Specifics on teaching such a course in London, as part of an international studies program, are discussed.
\end{abstract}




\section{INTRODUCTION}

It is important for physics departments to participate in a university's international and general education programs for reasons of visibility, attracting students to the major, maximal participation in degree offerings, and the advancement of general scientific literacy. Physicsbased general education courses typically include astronomy, physical science, ${ }^{1}$ courses that examine technology, ${ }^{2}$ and "physics for poets"3 courses. International education courses offered by a physics department are more difficult to come by even at a time when study abroad programs are seeing an increase in demand as students feel a need for a more global education. ${ }^{4}$

This paper describes a course taught by the author based on the problem of finding the longitude at sea. After a brief introduction to the problem, course planning advice, and a curriculum plan are presented, including several suggested activities and numerous references.

\section{THE LONGITUDE PROBLEM}

By the 1700s it was unfortunate that far-reaching expeditions were at great risk because there was no practical method of accurately determining one's navigational longitude. There are records of fateful expeditions both at land and sea ${ }^{5}$ due to this "longitude problem." Conditions at sea made this problem particularly serious, and many lives, ${ }^{6}$ property, and political prowess were lost. ${ }^{7}$ The longitude problem is well covered in both erudite ${ }^{5,8,9,10}$ and popular treatments. ${ }^{11,12,13,14,15}$

It was established that the simultaneous knowledge of one's remote (or "local") time and that at a fixed reference point ${ }^{16}$ would allow for the determination of the relative longitude. Knowing (or keeping) the time at the distant reference point (from afar) was the most problematic issue despite 
three known methods for doing so. The first involved using the motion of the Moon, the second involved keeping time with a portable chronometer, and the third used observations of the motion of Jupiter's moons. These were all sound solutions ${ }^{17}$ that lacked a practical implementation. Harsh conditions at sea ${ }^{18}$ where accurate navigation was the most critical posed the greatest challenges to a longitude solution. Observing Jupiter's moons at sea was impractical due to the difficulty in tracking them from a rocking ship. John Harrison ${ }^{9,11,19}$ spent a lifetime pursuing the chronometer approach, while a succession of astronomers employed at the Royal Greenwich Observatory $^{8,20,21}$ pursued the lunar approach. The interested parties were all competing for the substantial "longitude prize" offered by the British government in $1714 .{ }^{11}$ The chronometer approach eventually won, although the techniques using the Moon and Jupiter (on land) all became usable by the late 1700 s. The accuracy and ease of use of the marine chronometer caused it to become the dominant longitude-determining tool until worldwide time broadcasts in the early 1900s. The solution to finding longitude took the most convenient leap in the early 1990s, with the availability of the Global Positioning System (GPS).

The longitude problem was an outstanding worldwide problem in the 1700 s and was eventually solved using principles from physics and astronomy. We find it an appropriate topic for a college-level course for nonscience majors for the following reasons. A course whose topics include the longitude problem, celestial navigation, and timekeeping is an original offering of general interest to students. The longitude problem provides a framework for discussing a variety of scientific topics in support of understanding this problem (and its solution) from a historical and contemporary perspective. The longitude saga also presents an opportunity to demonstrate how science has worked successfully because it contains many of the essential elements seen in current scientific struggles, including a lag between a theory and experiment, pleas for funding, 
competition from other groups, originality, politics, experimental verification, dedication, and eventual triumph.

Initial course planning can be guided with the help of books by Sobel, ${ }^{11}$ Sobel and Andrewes, ${ }^{12}$ and Dash ${ }^{14}$ which provide concise and popular adaptations of the longitude problem. These books lack the scientific details needed for this course, which are amply supplied by Andrewes. ${ }^{5}$

A study of just the longitude problem alone will generally not fill a course consisting of approximately 40 lectures. The longitude problem has two very natural branches, one into celestial navigation ${ }^{22,23,24}$ and the other into the science of timekeeping. ${ }^{25,26,27,28}$ Our version of this course is divided into three units, which are detailed in the next section. The texts by Sobel, ${ }^{11}$ Sobel and Andrewes, ${ }^{12}$ Jespersen and Fitz-Randolph, ${ }^{25}$ Barnett, ${ }^{26}$ and Schlereth ${ }^{24}$ are required for the course and cover these three units.

\section{COURSE CURRICULUM}

\section{A. Preliminaries}

The need for basic mathematics throughout the course does not exceed the evaluation of trigonometric functions (with a calculator). Some necessary geometrical concepts include circles, triangles, spheres, lines, and angles, and their use in two- and three-dimensional space. Also needed are an understanding of scientific notation, analysis of graphs, the metric system, unit conversion, universal and astronomical constants, and the ability to evaluate simple expressions given numerical quantities. 
The goal of this section is to provide a guide and approximate schedule for implementing this course based on our experience. The estimates of the number of lectures needed are based on 50 min lectures.

\section{B. Unit 1: Understanding of the longitude problem}

We have identified five core ideas associated with understanding the longitude problem.

Discussions of these ideas are used to begin the course and are meant to thoroughly cover Ref. 11 with an emphasis on explaining the science therein.

The first, which generally serves as an introduction to the course, is a basic discussion of longitude and what events led to the establishment of the "Act of Longitude" by the British Parliament in 1714. ${ }^{5,11}$ The fateful journeys of Shovell and Anson ${ }^{5,11}$ were a consequence of longitude ignorance, and scurvy ${ }^{6}$ made haphazard navigation even more deadly (two lectures).

Second are the fundamental parameters that specify one's location on Earth, namely, latitude, longitude, and altitude. A thorough review of these parameters is typically needed, including some work with maps (three lectures). ${ }^{29,30,31}$

Third, there are many clues in nature that indicate one's latitude, but there are none for longitude. We find it easiest to introduce longitude by embarking on a detailed comparison between longitude and latitude, which involves a discussion of the Earth's placement and motion around the Sun, highlighting the "natural" lines of latitude, including the Equator and the Tropics of Cancer and Capricorn. Because there is no natural reference for longitude, an artificial one had to be invented, and it exists today at the Prime Meridian (three lectures). 
Fourth, in practice a reliable determination of longitude is based on the ability to keep accurate time. ${ }^{32}$ Earth rotates at $15 \%$, so for every $1 \mathrm{~h}$ one's local time differs from that at the Prime Meridian; their longitude is an additional $15^{\circ}$ east or west, as shown in Fig. 1 (two lectures).

Fifth, in the 1700s there were three methods for finding longitude; the problem was that no one had invented a practical method of implementing them with sufficient accuracy. ${ }^{33,34}$ As mentioned, using Jupiter's moons was quickly dismissed as a solution when at sea, leaving only two viable solutions. The first was the "Method of Lunars,",35 which uses the Moon to determine the time at the Prime Meridian. The second was keeping time at the Prime Meridian during a journey using a chronometer despite the harsh conditions onboard a typical ship. ${ }^{6,18}$ It is emphasized that both of these methods yielded the time at the Prime Meridian and nothing more. Local time was still needed, and both methods need multiplication of the difference between local and Prime Meridian time by $15^{\circ} / \mathrm{h}$ (two lectures).

\section{Unit 2: Celestial navigation}

Celestial navigation is a vast field, drawing heavily on astronomy, mathematics, and acquired skill. Often even the best references ${ }^{23,24,36}$ stress only the methodologies, not the underlying physical principles. ${ }^{22,37} \mathrm{~A}$ thorough discussion of both is well beyond the scope of this course, apart from a few general aspects of celestial navigation that are tied to the longitude problem.

\section{C1. The Sun}

The Sun is critically important in understanding the longitude problem, and a class discussion of its role can be started as follows. Suppose one is out at sea, with clear skies, and nothing visible but the featureless ocean, surrounded by the horizon in all directions. The instructor asks, "What 
single unique observation can be made to give some indication of one's location?" The answer (typically not obvious to students) is the observation of the Sun at its highest position in the sky, indicating "local noon." This observation appears over and over again in the longitude problem because it allows for an easy determination of one's local time (noon) and longitude if the time at the Prime Meridian is simultaneously known.

The Sun is also used to motivate two exercises in celestial navigation. First, the altitude angle of the Sun, $\boldsymbol{\alpha}$, can be measured ${ }^{38}$ at local noon, and its declination, $d$, at this time can be found using the Astronomical Almanac. ${ }^{39}$ From these two parameters, we can determine the latitude (in degrees) from $90^{\circ}-(\boldsymbol{\alpha}+d)$ as shown ${ }^{40}$ in Fig. 2. Second, because the Astronomical Almanac provides the geographic position ${ }^{23}$ of the Sun at all times of the day, the altitude angle taken at other (non-noon) times can be used to run through the process of celestial navigation. ${ }^{23,24}$ In particular, repeated altitude readings spaced, for example, at 1-h intervals ${ }^{41}$ can be used to narrow down one's position using Sumner's intercept method. ${ }^{23,24,37}$ This method allows for convergence between one's measured and actual position beginning with an educated guess. ${ }^{42}$ In class, this method provides a unique opportunity for working with maps and geometry in the context of navigation. Altitude angles of the Sun can be found (if not observed) from many Web-based resources (three lectures). ${ }^{43}$

\section{C2. Polaris}

For those in the Northern Hemisphere, Polaris provides a quick and easy exercise in celestial navigation. After finding a suitable horizon and ignoring a small wobble correction, ${ }^{24}$ the angular height of Polaris above the horizon approximates one's latitude. Using Polaris in this manner is easily illustrated in class using computer software ${ }^{44}$ by varying the latitude parameter from the 
North Pole to the Equator, while monitoring the plotted altitude of Polaris. We also do a night time exercise in observational astronomy, introducing the pointer stars of the Big Dipper to aid in finding Polaris. Finding angular distances between celestial objects is introduced using the technique eyeing one's fingers at arm's length ${ }^{45}$ because such distances are important in the method of lunars (see the following). Navigation with Polaris is concluded with a discussion of the technique of running the parallel (that is, maintaining constant latitude) used by early navigators (such as Columbus) with no means of finding longitude (one lecture).

\section{C3. The Moon}

The Moon played a particularly important role in the longitude problem because the method of lunars was the leading competitor to the chronometer solution for finding the longitude at sea. The method of lunars is complicated, and we discuss it in detail only as seems reasonable. The method is based on three facts. The first is that the Moon's motion across the sky is relatively quick. The Moon approximately moves one Moon diameter every $1 \mathrm{~h}\left(0.5^{\circ} / \mathrm{h}\right)$ with respect to the night time backdrop of the stars. Thus short-term repeated use of the Moon's motion for navigation is possible. The second is that the Moon's motion is irregular; its speed relative to a given star is not constant as time passes. This irregularity is shown in Fig. 3, where the rates of change in both right ascension and declination are seen to vary on a particular day by as much as $1^{\circ}$ in $24 \mathrm{~h}$. Available software ${ }^{44}$ will readily illustrate this effect. Third, the angular distance between the Moon and a given star (or planet) is unique for a given observation period.

The uniqueness of the nightly angular distance allows for the determination of the time at the Prime Meridian from a remote location, and creating tables linking angular distances with Prime Meridian times was the primary challenge of the astronomers at the Greenwich Observatory in 
the $1700 \mathrm{~s} .^{21}$ The topic of the Moon and its irregular motion is used in this course to discuss gravitation, including some numerical examples of Newton's universal of gravitation with a gentle discussion of the Sun-Earth-Moon system as a three-body problem. ${ }^{46}$ The relatively close proximity of the Moon to the Earth is used to discuss parallax and the need to correct for it, which is an important theme in celestial navigation. This concept is discussed again with Jupiter.

Isaac Newton is rightfully represented as a preeminent scientist with countless successes. His association with the longitude problem and the method of lunars is more unsettled. He expressed disappointment in the Moon as a navigational aid ${ }^{35}$ despite its brightness and universal familiarity. In addition, Newton had great difficulty in producing a theory to predict the Moon's motion, which was needed by astronomers to compile tables of its motion, so that navigators could execute the lunar method ${ }^{35}$ at sea. This difficulty is due to the intractability of the threebody problem, which is solved today using computers. ${ }^{47}$ Finally, Newton wrongly and emphatically declared that "nothing but astronomy is sufficient for this purpose [of finding longitude]" (see Ref. 5, p. 191) despite the fact that the marine chronometer eventually proved to be the most practical solution (three lectures).

\section{C4. Jupiter}

When visible at night, Jupiter is typically easy to find, and even a small telescope will reveal its four Galilean moons. ${ }^{48}$ The motion of Jupiter's moons was well known in the 1700 s, and it was thought that Jupiter could be used as the ultimate "navigational timekeeper" because the motion of the moons were well linked then, as now, ${ }^{39,49}$ to the time at the Prime Meridian. Such motions are easily illustrated using software. ${ }^{44}$ With these desirable properties, it is unfortunate that Jupiter was not a solution to the longitude problem at sea because a stable observation platform is 
needed to observe the moons. Jupiter is mentioned in this context due to its contribution in accurately finding longitude on land, which led to more accurate mapmaking ${ }^{12}$ and in testing Harrison's marine chronometers. ${ }^{5,12}$

Students will typically ask if precise Jupiter-moon timing events are universally observable from all points on Earth. This question can lead to an interesting exercise diagramming the parallax of Jupiter's moons, leading to a disagreement of such timings to a mere $0.5 \mathrm{~s}$ (for Io) from opposite points on the Earth (one lecture).

\section{Unit 3: The science of timekeeping}

The ability to keep accurate time was essential in solving the longitude problem and remains so in modern navigation, including the use of the global positioning system. A dedicated unit on timekeeping is essential. In this unit, many items found in a physics department's lecture demonstration inventory will be useful.

We begin with a general class discussion on time including a potentially spirited (but limited) discussion on the issue of "What is time?" before steering into the measurement and keeping of time. Feynman's ${ }^{50}$ ideas work as a start, and we follow with a discussion of time intervals from the very small (attosecond) to the very large (billions of years), physical phenomena associated with each, ${ }^{51}$ and the technology needed to observe a given time interval (two lectures).

Because this discussion alludes to extremely large and small time intervals, we begin a systematic analysis of natural timing phenomena that have allowed such time intervals to be measured. Celestial clocks (the Sun and Moon cycles), the mass/spring system, the simple 
pendulum, radioactive decay, and RC circuits are initially discussed. A discussion of these topics must be adapted to the instructional level desired ${ }^{1,2}$ (six lectures).

A discussion of "What is a clock?" begins with reference to mechanical clocks. ${ }^{2}$ As different types of clocks are discussed, four points are constantly emphasized. The first is whether or not a particular type of clock would work on a rocking ship. The second is the identification of the minimum components required by any clock, including an energy source, indicator, and escapement. ${ }^{52}$ The third is the identification of what environmental parameters would (adversely) affect the clock. Lastly, if timekeeping errors of a given clock are quantified, the subsequent error in longitude (at the Equator, for example) can be found and compared with the demands of the Act of Longitude. ${ }^{53}$

We begin with the mechanical periodicity achieved with verge-and-foliot-type clocks ${ }^{8,26,27,54}$ and an introduction to the concept of escapement. Pendulum clocks with a variety of escapement mechanisms are analyzed, which are tied to temperature compensation and the gridiron pendulum, which was perhaps Harrison's greatest achievement. ${ }^{5}$ If a pendulum is heated (or cooled), its length will increase (or decrease) leading to an increased (or decreased) period. The gridiron pendulum is a pendulum made of a combination of brass and steel rods cut in an inverse length ratio to the ratio of their expansion coefficients, as shown in Fig. 4. The system works because the thermal expansion is proportional to both the initial length and the expansion coefficient. Many short numerical examples are possible illustrating this effect in the context of thermal expansion (with an emphasis on brass and steel) (three lectures).

The innards of Harrison's first marine chronometer, $\mathrm{H} 1,{ }^{5,9,10}$ are analyzed, highlighting the torque free balances, the mass/spring timing element (at $1 \mathrm{~Hz}$ ), and Harrison's effort to minimize friction 
(in part) by exploiting rolling rather than sliding friction. The temperature compensation mechanism again uses a gridiron but indirectly compensates for the expanding or contracting balance lengths by adjusting the spring constant of the restoring spring. The idea of the massspring system is extended to a coiled mainspring, as illustrated in Harrison's fourth marine chronometer, $\mathrm{H} 4 .{ }^{5}$ A discussion of mechanical resonance ensues because Harrison's large clocks with small resonant frequencies often matched the rocking motion of the ship itself, causing undesirable coupling of the clock's internal motion to that of the ship. Harrison overcame this problem fortuitously with $\mathrm{H} 4$, which had a smaller more tightly wound mainspring that had a natural frequency much higher than any possible motion from the ship and thus was not affected by the ship's motion (three lectures).

Electronic clocks, with RC circuits at their core are discussed next, followed by the stabilization ability offered by the resonance effects of quartz crystals (two lectures).

Lastly, the atomic clock is discussed, highlighting the discovery of a time regulator (atomic energy levels) that is impervious to the common environmental parameters that so adversely impacted clocks of the past. The concepts of simple atom-photon absorption, transparency of an excitable gas, feedback, and light detectors are discussed. The need for a microwave generator, where the clock's time base ultimately comes from, is tied to the previous discussion of electronic clocks, though at a much higher frequency. Many of these components and concepts can be illustrated via a tour of a local research laboratory or advanced laboratory facility ${ }^{55}$ (three lectures). 


\section{E. Modern navigation: The global positioning system}

Although not as essential as those we have described, we feel obligated to discuss (near the end of the term) how the GPS system works because it presents a solution to the longitude problem for anyone (not just a few skilled navigators). We are amazed at the sheer quantity of physics

involved in the operation of a GPS,${ }^{56,57}$ which allows an instructor the freedom to handpick items for discussion. At minimum, the point is made that to solve the problem of navigation, the Earth had to be surrounded with approximately 30 satellites, all containing atomic clocks. The concept of measuring distance with time is discussed, as is simple two-dimensional triangulation, which is extended into three dimensions as needed. A bit of reflection is in order here as well as one of the first requirements of the GPS protocol is to return to the original challenge of the 1700s: To know what time is it at the Prime Meridian (two lectures).

\section{SUGGESTED ACTIVITIES}

We have developed several activities associated with the units both for courses taught on our campus during the regular term and in London, England during a study abroad summer term. They are briefly summarized here.

\section{A. On campus}

\section{A1. Navigation}

For an exercise in navigation using deduced reckoning, students are given pedometers and compasses. They then walk around campus starting at a known latitude and longitude. They are to record their headings and steps to later convert them to piecewise excursion distances, the 
totality of which is compared with the coordinates of their final observed destination (a spreadsheet can help with this calculation). Inherent errors in deduced reckoning are emphasized.

For a latitude/longitude tutorial, we have developed a Google map ${ }^{30}$ application, which allows students to find the latitude and longitude of any point in the world (such as their house or school) with a click of the mouse. ${ }^{31}$ Distances between points can be calculated as an exercise in evaluating trigonometric functions using the Haversine formula (or equivalent). ${ }^{37}$

If several sextants ${ }^{38}$ are available, an outside class activity can be done to estimate the altitude of the Sun (or other celestial object) and compare it with the Astronomical Almanac at that time. Students will find taking a shot with a sextant extremely difficult; most will not be able to shoot the bright Sun on a clear day. The difficulty of doing so from a rocking ship is emphasized. ${ }^{13}$ Simple protractor-plumb-bobs can be constructed and used with a nearby flag or light-pole.

\section{A2. Timing mechanisms}

Class sets of masses, springs, and pendulums can be used for in-class laboratory work on spring constants, pendulum lengths, masses, and their effects on oscillation periods.

A relaxation oscillator, built using a neon bulb and $\sim 100 \mathrm{~V}$ (dc) power source, can be used to illustrate of RC circuits and electronic timing. Breadboards with inexpensive integrated circuits such as the 555 timer $^{58}$ can be used to build a minimal electronic LED-flasher at 1-2 Hz to illustrate RC circuits and basic electronic clock construction.

A quartz crystal with a resonance frequency of $32.768 \mathrm{kHz}$ can be wired in parallel to a 1000 resistor, then driven with an external oscillator while monitoring the voltage drop across the 
resistor using an oscilloscope. A very sharp resonance response will be observed with a

$Q \approx 100000$.

An in-class radioactive decay activity can be run by issuing groups of students $\sim 15$ dice cubes.

Rolling them all at once and extracting those that show a predetermined number (a "decay") can simulate the statistics observed in radioactive decay.

\section{A3. Observational astronomy}

Two night time activities are possible. The first is to use a small telescope to observe Jupiter and its moons with some coordination to monthly activity tables. ${ }^{49}$ The second (for those in the northern hemisphere) is to find Polaris and estimate one's latitude using a sextant or protractorplum-bob.

\section{A4. Modern navigation}

Bare GPS receivers are available for under $\$ 100,{ }^{59}$ which send a data-stream containing universal time, longitude, latitude, and altitude, readily captured using the serial-port of a computer.

A project to highlight the process of invention is the digital compass, which can be constructed using LEDs to indicate the direction of travel. ${ }^{60}$

\section{B. In London}

Many universities have study abroad programs, the curriculum of which might be outsourced or led by the university's own faculty. If faculty led, there is an opportunity to create this class for a London, England-based course, where the longitude saga comes to life. There are artifacts of the longitude problem scattered throughout the city. The major attraction is the Greenwich 
Observatory, which represents the entire longitude struggle in one location, including all of Harrison's marine chronometers. Salisbury Cathedral contains a working $600+$ year old vergeand-foliot clock. St. John's Cathedral in Hampstead Heath is the burial place of Harrison, and the inscriptions on both sides of the large tomb are well worth studying. Westminster Abbey memorializes many important scientists of the time, including Newton. A memorial can be found for Harrison, as can one of the largest and ugliest ${ }^{8}$ for Shovell. The Worshipful Co. of Clockmakers Museum houses perhaps the densest collection of working mechanical clocks in the world, where students can watch the movement of dozens of different clocks. A small memorial to Harrison can also be found there. Red Lion Square holds a blue marker plaque dedicated to Harrison, highlighting where he worked and lived. The Wallace Collection houses dozens of large mechanical clocks, allowing students to observe many large gridiron pendulums and other temperature compensation mechanisms. Hogarth's famous engraving entitled "Rakes Progress" can be studied there; a detail suggests the prevailing thoughts about the longitude problem in the 1720s. The Science Museum of London houses one of Harrison's early land-based clocks and the scientific instruments of King George III, who was an active amateur scientist and a staunch supporter of Harrison. The British Museum houses a world-class horology exhibit.

Spain, Portugal, and France also played very prominent roles in the history of the longitude problem, and it would be possible to find connections for this class in international studies programs based in these countries as well.

\section{CONCLUSIONS}

We have described the essential elements of an international or general education class on the longitude problem from the 1700 s to today. The problem is full of physics and astronomy-based 
topics that may be adapted to a non-science-major level of instruction in the context of a historical struggle to solve a worldwide problem.

Two underlying themes of the course are also suggested. The first is how incremental advances in technology continually change our priorities. ${ }^{5}$ Longitude, for example, is now found by simply pressing a button ${ }^{5}$ on a GPS receiver. Second, Harrison was not a scientist but a clockmaker and carpenter, yet he found a solution that eluded the best scientists in history, highlighting a case where steadfast dedication, against the odds, led to a successful outcome.

The author has taught such a class and has adapted it to a group of $\sim 20$ students for an international studies program in London and $\sim 50$ students in a regularly offered campus-based general education course. Both courses enroll students with mostly nonscience backgrounds and degree interests, and the author has not experienced difficulty in presenting the longitude problem to them using all of the ideas suggested in this paper. A final class project consists of students proposing a solution to a worldwide problem of their choosing; those committed to their proposal are encouraged to submit it for possible funding. ${ }^{61}$

\section{ACKNOWLEDGMENTS}

The author is indebted to D. Doty, M. Moelter, R. Echols, J. Keller, and W. J. H. Andrewes for many useful discussions. Thanks are due to J. Battenburg for the London opportunity in 2008 and S. Kim, M. Armstrong, and the 2008 London Study students for their enthusiasm. London-based instructor J. Makey is thanked for his exquisite knowledge of London, and the author is indebted to Jonathan Betts, Senior Horologist at the Greenwich Observatory, for a dedicated tour of the facilities. Thanks are also due to the very professional and hospitable staff at the London-based 
Foundation for International Education ${ }^{62}$ for hosting our group. Finally, thanks are due to the anonymous referees for their comments that helped to clarify many aspects of this paper. 


\section{REFERENCES}

1. P. G. Hewitt, Conceptual Physics, 10th ed. (Addison-Wesley, New York, 2008).

2. L. A. Bloomfield, How Things Work: The Physics of Everyday Life, 3rd ed. (Wiley, New York, 2005).

3. Courses based on the following texts: R. H. March, Physics for Poets, 4th ed. (McGrawHill, New York, 1995)

B. R. Greene, The Elegant Universe (Norton, New York, 2003)

S. W. Hawking, A Brief History of Time (Batam, New York, 1996)

B. M. Bryson, A Short History of Nearly Everything (Broadway, New York, 2003).

4. B. McMurtrie, "Study-abroad numbers continue to climb, trips are shorter, report says," Chronicle Higher Educ. 54(12), A36 (2007).

5. The Quest for Longitude: The Proceedings of the Longitude Symposium Harvard University, Cambridge, Massachusetts, edited by W. J. H. Andrewes (Collection of Historical Scientific Instruments, Cambridge, MA, 1993).

6. S. R. Brown, Scurvy: How a Surgeon, a Mariner, and a Gentleman Solve the Greatest Medical Mystery of the Age of Sail (Viking, New York, 2003).

7. The need for accurate longitude determination affected cartographers and maritime and terrestrial navigators alike. Although the longitude problem generally focuses on maritime navigators, the accuracy of the best maps suffered greatly as well (Ref. 63), as highlighted in the journeys of LaSalle and Shovell (Ref. 5).

8. D. Howse, Greenwich Time and the Discovery of Longitude (Oxford U. P., Oxford, 1980). 
9. J. Betts, Time Restored: The Harrison Timekeepers and R. T. Gould, the Man Who Knew (Almost) Everything (Oxford U. P., Oxford, 2006).

10. R. T. Gould, The Marine Chronometer: Its History and Development, 2 nd revised ed. (Holland, Suffolk, 1960).

11. D. Sobel, Longitude: The True Story of a Lone Genius Who Solved the Greatest Scientific Problem of His Time (Walker, New York, 2007).

12. D. Sobel and W. J. H. Andrewes, The Illustrated Longitude: The True Story of a Lone Genius Who Solved the Greatest Scientific Problem of His Time (Walker, New York, 2003).

13. NOVA/Public Broadcasting Service, NOVA: Lost at Sea-The Search for Longitude (DVD) (WGBH Boston, Boston, 2008).

14. J. Dash, The Longitude Prize (Farrar, Straus and Giroux (BYR), New York, 2000).

15. C. Sturridge (director), Longitude (ITV DVD, London, 1999).

16. The familiar reference for $0^{\circ}$ longitude at the Prime Meridian in Greenwich, England, was not fully recognized until the late 1800 s.

17. See the discussions of "nutty" solutions to the longitude problem in Refs. 5,11.

18. P. Kirsch, The Galleon: The Great Ships of the Armada Era (Naval Institute, Annapolis, MD, 1990).

19. J. Betts, Harrision, 2nd ed. (National Maritime Museum, London, 2007).

20. See: www.nmm.ac.uk.

21. English astronomers were not the only ones to carefully study the Moon's motion. Tobias Mayer and Leonhard Euler contributed greatly in this area. 
22. J. A. Van Allen, "Basic principles of celestial navigation,” Am. J. Phys. 72, 1418-1424 (2004).

23. M. Kelch, Simplified Celestial Navigation (Haessner, New York, 1975).

24. H. Schlereth, Celestial Navigation in a Nutshell (Sheridan House, New York, 2000).

25. J. Jespersen and J. Fitz-Randolph, From Sundials to Atomic Clocks: Understanding Time and Frequency, 2nd revised ed. (Dover, New York, 1999).

26. J. E. Barnett, Time's Pendulum: From Sundials to Atomic Clocks, The Fascinating History of Timekeeping and How Our Discoveries Changed the World (Harcourt Brace/Harvest Book, New York, 1999).

27. P. Hood, How Time Is Measured, 2nd ed. (Oxford U. P., Oxford, 1969).

28. H. J. Cowan, Time and Its Measurements: From the Stone Age to the Nuclear Age (World, Cleveland, 1958).

29. R. A. Rutherford, Where on Earth?: Understanding Latitude and Longitude (Walch Education, Portland, ME, 1989).

30. See: maps.google.com, which contains a programming interface allowing for custom programs that use the map functionality.

31. See: ocean.physics.calpoly.edu/longitude/map.php. The source to this Google-map application is available by contacting the author.

32. In principle, longitude (and latitude) can be kept by maintaining a piecewise log of distance and heading relative to a known reference point, which is the essence of "deduced reckoning." This method continues to be an important baseline in navigation even though it is plagued by systematic errors, at best giving only a sense of one's 
location during a long journey. Deduced reckoning errors on transoceanic voyages of hundreds of miles were typical in the 1700s (Ref. 5).

33. We see many parallels between finding longitude and that of producing controlled fusion. Both have sound scientific backings; the challenge is in the implementation.

34. For 18th century views on finding longitude, we suggest studying an engraving by William Hogarth, Plate 8, titled, “A Rake's Progress” as shown in Ref. 12, p. 105.

35. N. Kollerstrom, Newton's Forgotten Lunar Theory, His Contribution to the Quest for Longitude: Includes Newton's Theory of the Moon's Motion (Green Lion, Santa Fe, 2000).

36. N. Bowditch, The American Practical Navigator, 5th ed. (Edmund M., Blunt, New York, 1821).

37. H. Umland, “A short guide to celestial navigation,” www.celnav.de.

38. See the Mark 3 sextant at www.davisnet.com.

39. Nautical Almanac Office of the United States Naval Observatory, The Astronomical Almanac for the Year (Department of the Navy, Washington, D.C., 2008).

40. D. H. Sadler, Man is Not Lost: A Record of Two Hundred Years of Astronomical Navigation with The Nautical Almanac, 1767-1967 (National Maritime Museum, London, 1968).

41. H. Schlereth, Commonsense Celestial Navigation (Regnery, Washington, D.C., 1975).

42. A. J. Deutsch, "The probability distribution around a fix in celestial navigation," Am. J. Phys. 13, 379-383 (1945).

43. For a web-based calculator of the Sun's altitude, see: www.learn.londonmet.ac.uk/packages/daymedia/axel/sky/sunpath_calc.html. 
44. See: www.starrynight.com/.

45. P. H. Harrington and E. Pascuzzi, Astronomy for All Ages: Discovering the Universe Through Activities for Children and Adults, 2nd ed. (Globe Pequot, Guilford, CT, 2000).

46. M. E. R. Walford, “An experimental measurement of longitude,” Phys. Educ. 32, 46-48 (1997).

47. See: www.math.uu.nl/people/wepster/ldtab.html.

48. A simple $20 \mathrm{~s}$ exposure of Jupiter with a digital SLR camera will reveal its moons.

49. See the magazines Sky and Telescope and Astronomy.

50. R. P. Feynman, R. B. Leighton, and M. Sands, The Feynman Lectures on Physics (Addison-Wesley, New York, 1964), Vol. 1.

51. D. Labrador, "From instantaneous to eternal," Sci. Am. 287(3), 56-57 (2002).

52. The energy source is what keeps a clock running (for example, a falling weight, coiled spring, or electricity). An indicator allows one to learn the time from a clock (for example, dial and hands, digital display, or radio signals). An escapement allows for periodic release of energy from the energy source, typically setting the smallest time base for a clock (for example, balance and spring, pendulum, or transistor gate). See Ref. 26, p. 147.

53. Harrison's fourth marine chronometer, $\mathrm{H} 4$, lost 0.78 s/day over a 60 -day round-trip voyage. This loss is equivalent to an error in longitude of $0.033^{\circ} /$ day or approximately 14 miles (at the Equator) upon return.

54. A "verge and foliot" escapement is perhaps the earliest mechanical escapement known. It consists of a saw-toothed wheel that is turned by a weighted rope wrapped around its axis. As it turns, it is periodically stopped by two pallets that alternately engage the teeth 
on opposite diameter points of the wheel. The pallets are attached to a vertical rod (the verge) that is oriented along the engagement diameter. A horizontal bar (the foliot) it attached to the top of the verge rod, forming a T-shape, characteristic of this type of escapement. Weights hanging from the foliot allow for adjustments to its moment of inertia, setting the periodicity of the escapement. See Ref. 26, p. 65.

55. An advanced physics laboratory class on campus might have an optical pumping apparatus available, which contains many of the essential elements of an atomic clock. See the Optical Pumping apparatus available from TeachSpin www.teachspin.com, for example.

56. N. Ashby, "Relativity and the global positioning system," Phys. Today 55(5), 41-47 (2002).

57. T. Logsdon, Understanding the Navstar: GPS, GIS, and IVHS, 2nd ed. (Van Nostrand Reinhold, New York, 1995).

58. See All Electronics Corporation, Part numbers: LM555, PB400, BST41, JW140, LED-1, www.allelectronics.com.

59. See: GPS-08234 at www.sparkfun.com.

60. See the 1490 digital compass at www.imagesco.com.

61. See the Gates Foundation at www.gatesfoundation.org or the XPrize foundation at www.xprize.org.

62. Foundation for International Education, 114 Cromwell Rd., London SW7 4ES, fie.org.uk/.

63. L. A. Brown, The Story of Maps (Dover, New York, 1980). 


\section{FIGURES}

\section{Figure 1}

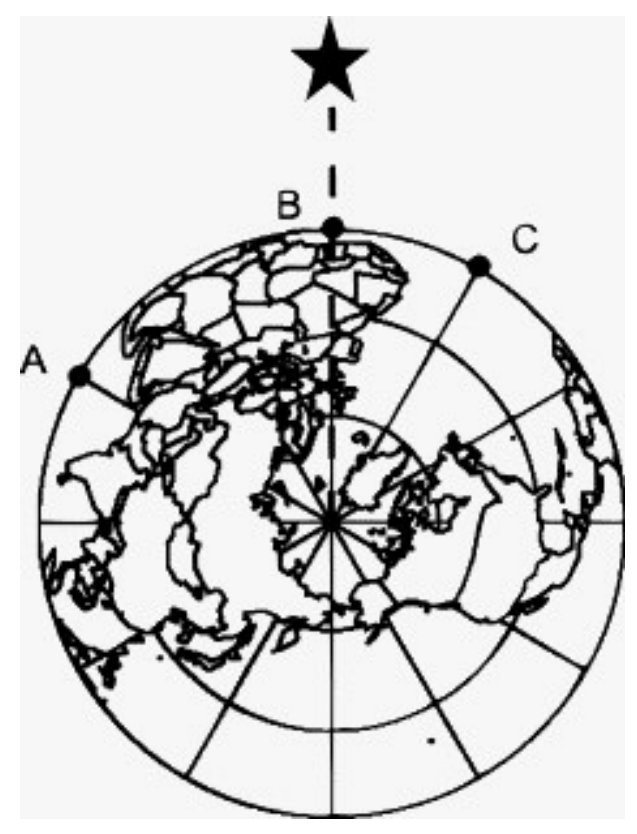

Fig. 1. A view of Earth from above the North Pole, which rotates counterclockwise as shown. The line from the North Pole through point B represents the Prime Meridian, at which it is currently noon because the Sun (star symbol) is directly overhead. Noon has already occurred for an observer at A; hence, they are east of the Prime Meridian. Noon has yet to occur for an observer at C; they are west of the Prime Meridian. The time difference between locations A and B (or B and C) times the Earth's rotation rate of $15^{\circ} / \mathrm{h}$ yields the relative longitude. Finding the longitude requires simultaneous knowledge of one's local time and that at the Prime Meridian. Note that this method is independent of the observer's latitude. 


\section{Figure 2}

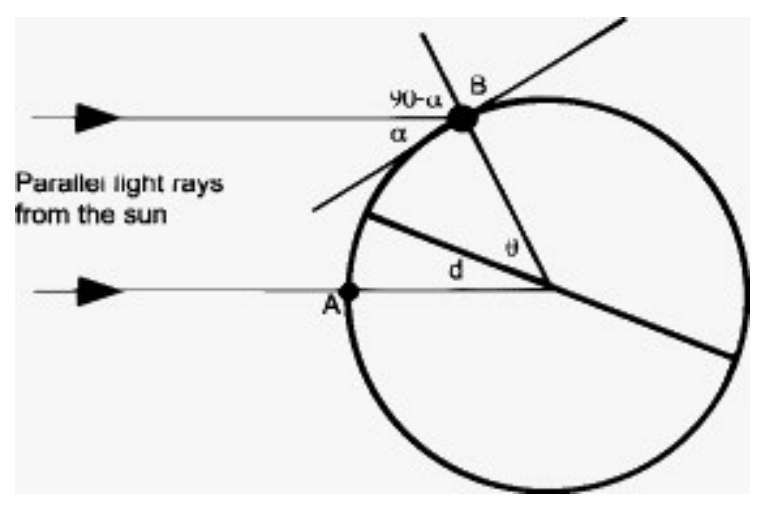

Fig. 2. The Earth and Equator are shown. Point A represents the position of the Sun with a declination $d$, as determined from the Astronomical Almanac (Ref. 39) at this particular date and time. Point B represents the location of an observer with latitude $\theta$. Because it is local noon at B, the Sun is over B's line of longitude, which corresponds to the outline of the Earth. The line tangent to the Earth at B is the local horizon, so $\boldsymbol{\alpha}$ is the observed altitude angle of the Sun. It follows that $90-\boldsymbol{\alpha}=d+\boldsymbol{\theta}$, allowing $\theta$ (latitude) to be determined from the other known quantities. 


\section{Figure 3}

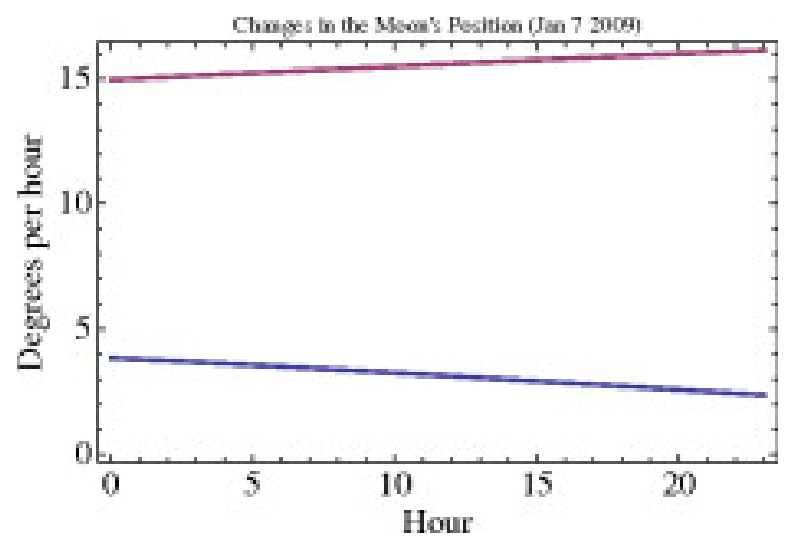

Fig. 3. Evidence of the Moon's irregular motion using data obtained directly from Ref. 39 for January 7 , 2009. The curves are time derivatives of the Moon's right ascension (upper curve) and declination (lower curve) over $24 \mathrm{~h}$, both of which contribute to the Moon's net velocity. Variations by as much as $1 \% / 24 \mathrm{~h}$ in each occurred on this particular day. 


\section{Figure 4}

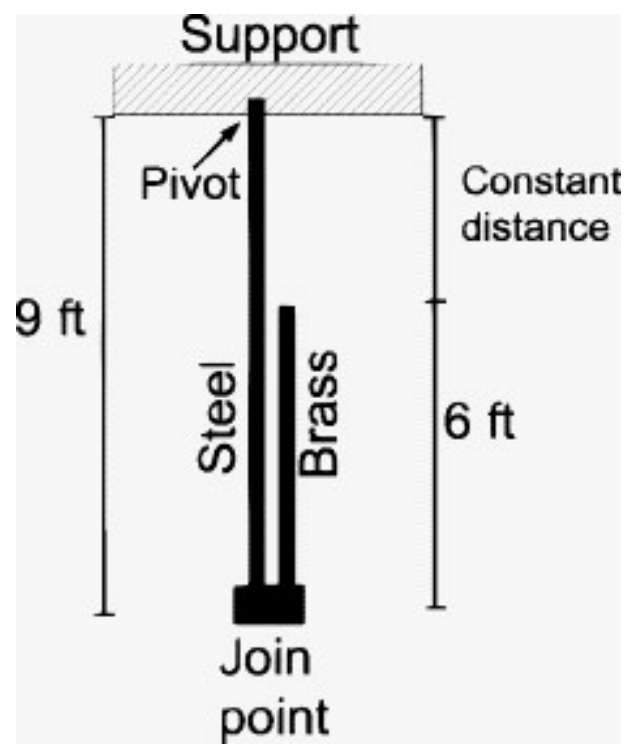

Fig. 4. The simplest gridiron made of brass (gray) and steel (black) rods. Here the $9 \mathrm{ft}$ steel rod is attached to a support from above and fixed to a $6 \mathrm{ft}$ brass rod at its bottom. If the system is heated (cooled), the brass will expand (contract) more than the steel by a factor of $\approx 3 / 2$. Because the rod lengths are cut by the inverse ratio of $6 / 9$, the longer steel rod will expand downward by the same distance that the shorter brass rod expands upward. The distance between the support and top of the brass rod will thus remain approximately constant as a function of temperature. This configuration shows that it is possible to create a separation distance with a minimal dependence on temperature. A pendulum bob, for example, could be attached to the top of the brass rod. Illustrations of more practical designs for pendulums can be found in Ref. 5. 